\title{
Electrochemical strain microscopy time spectroscopy: Model and experiment on $\mathrm{LiMn}_{2} \mathrm{O}_{4}$
}

Hugues-Yanis Amanieu', Huy N. M. Thai', Sergey Yu. Luchkin', Daniele Rosato', Doru C. Lupascu’, MarcAndré Keip' , Jörg Schröder', and Andrei L. Kholkin'

Citation: Journal of Applied Physics 118, 055101 (2015); doi: 10.1063/1.4927747

View online: http://dx.doi.org/10.1063/1.4927747

View Table of Contents: http://aip.scitation.org/toc/jap/118/5

Published by the American Institute of Physics

\section{Articles you may be interested in}

$\mathrm{Li}$ transport in fresh and aged LiMn2O4 cathodes via electrochemical strain microscopy Journal of Applied Physics 118, 072016072016 (2015); 10.1063/1.4927816

Imaging space charge regions in Sm-doped ceria using electrochemical strain microscopy Journal of Applied Physics 105, 201602201602 (2014); 10.1063/1.4901102

Characterization of LiMn2O4 cathodes by electrochemical strain microscopy Journal of Applied Physics 108, 113106113106 (2016); 10.1063/1.4943944

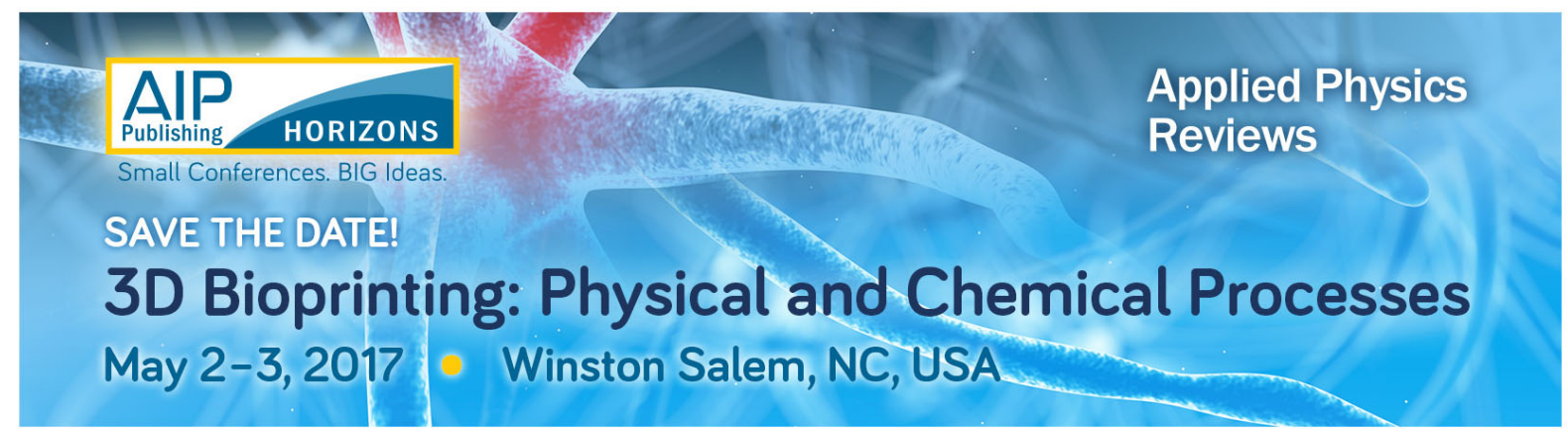




\title{
Electrochemical strain microscopy time spectroscopy: Model and experiment on $\mathrm{LiMn}_{2} \mathrm{O}_{4}$
}

\author{
Hugues-Yanis Amanieu, ${ }^{1,2, a), b)}$ Huy N. M. Thai, ${ }^{3, b), c)}$ Sergey Yu. Luchkin,, ${ }^{4, b), d)}$ \\ Daniele Rosato, ${ }^{1, \mathrm{e})}$ Doru C. Lupascu, ${ }^{2, \mathrm{f})}$ Marc-André Keip, ${ }^{5, \mathrm{~g})}$ Jörg Schröder, ${ }^{3, \mathrm{~h})}$ \\ and Andrei L. Kholkin ${ }^{4,6, i)}$ \\ ${ }^{1}$ Robert Bosch GmbH, Robert Bosch Platz 1, 70839 Gerlingen Schillerhoehe, Germany \\ ${ }^{2}$ Institute of Materials Science, University of Duisburg Essen, Universitätsstr. 15, 45141 Essen, Germany \\ ${ }^{3}$ Institute of Mechanics, University of Duisburg Essen, Universitätsstr. 15, 45141 Essen, Germany \\ ${ }^{4}$ Department of Physics \& CICECO, University of Aveiro, 3810193 Aveiro, Portugal \\ ${ }^{5}$ Institute of Applied Mechanics (CE), Chair I, University of Stuttgart, Pfaffenwaldring 7, 70569 Stuttgart, \\ Germany \\ ${ }^{6}$ Institute of Natural Sciences, Ural Federal University, Ekaterinburg 620000, Russia
}

(Received 28 April 2015; accepted 21 July 2015; published online 6 August 2015)

\begin{abstract}
Electrochemical Strain Microscopy (ESM) can provide useful information on ionic diffusion in solids at the local scale. In this work, a finite element model of ESM measurements was developed and applied to commercial lithium manganese (III,IV) oxide $\left(\mathrm{LiMn}_{2} \mathrm{O}_{4}\right)$ particles. ESM time spectroscopy was used, where a direct current (DC) voltage pulse locally disturbs the spatial distribution of mobile ions. After the pulse is off, the ions return to equilibrium at a rate which depends on the Li diffusivity in the material. At each stage, Li diffusivity is monitored by measuring the ESM response to a small alternative current (AC) voltage simultaneously applied to the tip. The model separates two different mechanisms, one linked to the response to DC bias and another one related to the AC excitation. It is argued that the second one is not diffusiondriven but is rather a contribution of the sum of several mechanisms with at least one depending on the lithium ion concentration explaining the relaxation process. With proper fitting of this decay, diffusion coefficients of lithium hosts could be extracted. Additionally, the effect of phase transition in $\mathrm{LiMn}_{2} \mathrm{O}_{4}$ is taken into account, explaining some experimental observations. (C) 2015 AIP Publishing LLC. [http://dx.doi.org/10.1063/1.4927747]
\end{abstract}

\section{INTRODUCTION}

Commercially available Li-ion batteries do not deliver a long life cycle for high cycling rates needed for heavy duty applications, such as for the automotive industry. ${ }^{1}$ Micromechanical effects associated with cycling significantly contribute to degradation. Lithium intercalation and de-intercalation result in volume expansion and contraction as well as in phase changes in active electrode particles. This micromechanical fatigue is one of the origins of capacity loss in many materials. ${ }^{2}$ Therefore, a thorough understanding of functional properties and degradation mechanisms is required at the microscale.

Conventional electrochemical methods such as impedance spectroscopy, galvanostatic, or potentiostatic intermittent titration techniques can hardly be used to study functional properties at the local scale. A relatively new Atomic Force Microscopy (AFM) based method,

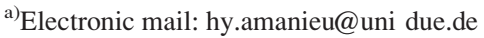

${ }^{b)}$ H. Y. Amanieu, H. Thai, and S. Luchkin contributed equally to this work.

${ }^{c)}$ Electronic mail: huy.thai@uni due.de

d)Electronic mail: luchkin@ua.pt

${ }^{\text {e)} E l e c t r o n i c ~ m a i l: ~ d a n i e l e . r o s a t o @ d e . b o s c h . c o m ~}$

${ }^{\mathrm{f}}$ Electronic mail: doru.lupascu@uni due.de

${ }^{\mathrm{g})}$ Electronic mail: marc andre.keip@mechbau.uni stuttgart.de

h)Electronic mail: j.schroeder@uni due.de

${ }^{i)}$ Electronic mail: kholkin@ua.pt
}

Electrochemical Strain Microscopy (ESM), can tackle this issue. It can probe ionic properties within micrometric particles. $^{3}$

ESM is based on the detection of surface displacement caused by ionic motion under an AFM tip. More precisely, an external alternative current (AC) voltage is applied between a conducting AFM tip operating in contact mode and the sample, acting as counter electrode. The AFM measures the alternating displacement of the cantilever. There can be several causes, including but not limited to ionic motion; for example, space charge, flexoelectricity, and dipolar contribution depending on the material properties. ${ }^{4}$ It has been proposed to perform time spectroscopy measurements in order to characterize ionic motion only. ${ }^{5}$ This method consists of application of a direct current (DC) pulse before acquiring the $\mathrm{AC}$ displacements. As ions are moving towards (outwards) the tip, the ESM signal increases (decreases), respectively. Therefore, the variation of the ESM response after the DC pulse is governed by the ionic diffusion. At this stage, local electronic space charge relaxation (MaxwellWagner relaxation) also takes place. However, this process is typically much faster than the ionic diffusion unless injected charges are trapped. ${ }^{6} \mathrm{In}_{\mathrm{Li}_{x}} \mathrm{Mn}_{2} \mathrm{O}_{4}$, with a static dielectric permittivity of $\approx 10{ }^{7}$ an electrical conductivity is $10^{4} 10^{2} \mathrm{~S} \mathrm{~m}^{1}$ (Refs. 1 and 8) and a lithium diffusion coefficient is $10^{16} 10^{13} \mathrm{~m}^{2} \mathrm{~s} \mathrm{~s}^{1,}$, the space charge 
relaxation time is $10^{8} 10^{6} \mathrm{~s}$, while the diffusion relaxation time for the ESM probed volume is $10^{3} 10^{2} \mathrm{~s}^{9}$ Furthermore, space charge relaxation follows an exponential law while diffusion relaxation typically obeys a power law.

An electrochemical-mechanical model was proposed by Morozovska et $a l^{3}$ to explain the influence of the ionic motion under ESM excitation. It was assumed that the outof-plane displacement in the frequency domain is proportional to the local change of lattice volume induced by the change of concentration below the tip as follows:

$$
u_{z}(0, \omega) \propto-(1+\nu) \Omega_{\mathrm{Li}}\left\langle\delta c_{\mathrm{Li}}\right\rangle,
$$

where $\Omega_{\mathrm{Li}}$ is the molar volume expansion induced by lithiation directly related to Vegard's tensor $\beta$ through Avogadro's number, $\nu$ is Poisson's ratio, and $\left\langle\delta c_{\mathrm{Li}}\right\rangle$ is the average change of lithium ion concentration below the tip. They suggested that the produced electrochemical strain is mediated by the local transport mechanisms controlled (among others) by the ionic diffusivity $D_{d}$. Later Morozovska et al. ${ }^{10}$ used this concentration-change based model in order to uncover the image formation mechanisms for systems with ion-blocking electrodes and without local electroneutrality. They computed values for two lithium host materials: $\mathrm{LiCoO}_{2}$ and $\mathrm{LiMn}_{2} \mathrm{O}_{4}$, and showed a displacement much lower than $1 \mathrm{pm}$ for frequencies above $10^{4} \mathrm{~Hz}$ even though a relatively high diffusion coefficient of $10{ }^{13} \mathrm{~m}^{2} \mathrm{~s}{ }^{1}$ was used. Based on the same philosophy, another study was reported by the same group, ${ }^{11}$ aimed at exploring time and length scales of the dynamic response. They quantified that the maximum vertical displacement for an $\mathrm{AC}$ excitation at $10^{6} \mathrm{~Hz}$ is again much below $1 \mathrm{pm}$ for a diffusion coefficient of $10{ }^{14} \mathrm{~m}^{2} \mathrm{~s}{ }^{1}$. Besides, they found a mean oscillation path for the ions smaller than the distance between two interstitials, meaning that the diffusion-based model cannot be applied in this case.

This effect can be compared to traditional impedance spectroscopy where it is recognized that a signal originating from a high frequency excitation $(>100 \mathrm{kHz})$ does not give information on the ionic transport mechanism as they are too slow but are rather dependent on capacitive and other electronic effects. ${ }^{12}$ However, ESM signal generation was experimentally demonstrated at high frequencies $200400 \mathrm{kHz}$, with a typical relaxation process that can be probed using time spectroscopy. $9,13,14$

In this work, we aim at simulating ESM time spectroscopy (ESM-TS) measurements on a single $\mathrm{LiMn}_{2} \mathrm{O}_{4}$ particle. While the dynamic signal (AC) of ESM excitation in the $0.11 \mathrm{MHz}$ range is too quick to impact ionic transport, the DC pulses used in ESM-TS measurements are long enough to produce a local concentration change below the tip. Considering time domain, Morozovska et $\mathrm{al}^{3}{ }^{3}$ also proposed an analytical model for the out-of-plane response induced by lithium redistribution under the DC pulse. They found a power law for the surface relaxation in the case of a concentration-controlled process (ion-blocking electrode) as follows:

$$
u_{z}\left(0, t \gg t_{0}\right) \approx-(1+\nu) \frac{\beta \phi_{0} R_{\mathrm{tip}}^{2} t_{0}}{6 \eta \sqrt{ } \pi D_{0} t^{3 / 2}},
$$

where $R_{\text {tip }}$ is the tip contact radius, $\phi_{0}$ is the applied voltage, $t_{0}$ is the length of the DC pulse, $D_{0}$ is the diffusion coefficient of $\mathrm{Li}$, and $\eta$ is a coefficient related to the ohmic behavior of the tip contact.

$\mathrm{Li}_{x} \mathrm{Mn}_{2} \mathrm{O}_{4}$ experiences two phase changes upon lithiation and delithiation, ${ }^{15-17}$ one between $\lambda-\mathrm{MnO}_{2}$ and $\mathrm{Li}_{0.5} \mathrm{Mn}_{2} \mathrm{O}_{4}$ and another one between $\mathrm{Li}_{0.5} \mathrm{Mn}_{2} \mathrm{O}_{4}$ and $\mathrm{LiMn}_{2} \mathrm{O}_{4}$. This translates into irregularities in the Gibbs free energy evolution of the material upon change of lithium concentration, ${ }^{18}$ leading to a phase growing into another one instead of having a smooth lithium concentration gradient across the particles. Bohn et al. ${ }^{19}$ developed a chemicalmechanical coupling model taking into account this irregularity by using an effective diffusion coefficient, denoted $D_{\text {eff }}$ and introduced in Section III A.

The present work is based on reusing the formal description of Bohn et al., ${ }^{19}$ which is very similar to previous works done on ESM modeling, ${ }^{3,10,20}$ and will also compare the influence of $D_{\text {eff }}$ on the ESM signal. The objective is to model the time evolution of lithium concentration, surface displacement, and the ESM signal below the tip during and after DC pulses. Our model will approach the philosophy of Morozovska et al. ${ }^{10}$ in the sense that only ion-blocking electrodes will be considered. However, local electroneutrality will be preserved for simplicity. The main difference with previous simulation works ${ }^{10,20}$ is that the dynamic (AC) mechanical response will be estimated differently and irregularities in the Gibbs free energy will be considered. Only time domain response will be examined and compared to previous analytical work (Ref. 3). As such, a method to study ionic transport from the relaxation of the ESM signal in the time domain is proposed in this work.

\section{EXPERIMENTAL}

$\mathrm{LiMn}_{2} \mathrm{O}_{4}$-based cathode materials were extracted from fresh commercially available electrochemical cells at $0 \%$ state of charge (SoC), $50 \% \mathrm{SoC}$, and $100 \% \mathrm{SoC}$ as well as from a cell aged at 16C-rate down to a state of health of $80 \%$ and opened at $0 \%$ SoC. Cross-sections of the cathodes were prepared and finely mechanically polished with final ionbeam polishing. The whole procedure is thoroughly detailed elsewhere. ${ }^{21}$

ESM measurements were performed using a commercial AFM (Solver Next, NT-MDT, Russia) working at room temperature under ambient conditions. $\mathrm{LiMn}_{2} \mathrm{O}_{4}$ cathode samples were grounded via the $\mathrm{Al}$ current collector serving as a counter electrode. Voltage was applied to a Pt/Ir coated cantilever with a stiffness of about $5 \mathrm{~N} / \mathrm{m}$ and $105 \mathrm{kHz}$ resonance frequency. The probing AC-voltage was $3 \mathrm{~V}$ in amplitude and $1 \mathrm{MHz}$ frequency. The response was measured by the internal lock-in amplifier of the microscope. Time spectroscopy ESM measurements ${ }^{9}$ were performed after application of a $10 \mathrm{~ms}$ rectangular $10 \mathrm{~V}$ DC pulse. Voltage spectroscopy ESM measurements ${ }^{22}$ were performed by applying $10 \mathrm{~ms}$ DC pulses of increasing and decreasing amplitude with 100 pulses per cycle. ESM response was acquired between the pulses in the DC-off state. 


\section{MODEL DESCRIPTION}

\section{A. Thermodynamical description}

In this section, a body of $\mathrm{LiMn}_{2} \mathrm{O}_{4}$ material is described in order to model its response to the DC pulse employed in time spectroscopy.

According to the Gibbs free energy described in Appendix $\mathrm{A},{ }^{23}$ we obtain the mechanical stress as (see Eq. (A6) in Ref. 23)

$$
\boldsymbol{\sigma}=\mathbb{C}\left(\varepsilon-\frac{1}{3} \Omega_{\mathrm{Li}}\left(c_{\mathrm{Li}}-c_{0}\right) \mathbf{1}\right),
$$

where $\mathbf{1}$ is the second-order identity tensor (Kronecker delta), $\mathbb{C}$ is the elastic tensor, $\varepsilon$ is the total strain, $c_{\mathrm{Li}}=N / V_{0}$ is the concentration of lithium ions, with $N$ being the number of lithium ions per unit volume $V_{0}$, and $c_{0}$ is the initial concentration. $\Omega_{\mathrm{Li}} / 3$ is the isotropic approximation of the partial molar volume. ${ }^{19}$ It is constant over the range of the intercalation and it is small enough in $\mathrm{LiMn}_{2} \mathrm{O}_{4}$ to consider smallstrain theory (contrary to, e.g., silicon anodes ${ }^{24}$ ). It is directly related to Vegard's tensor. Other electromechanical couplings were disregarded as Vegard's deformation dominates in a time domain. ${ }^{4}$

The electrochemical potential of lithium ions is derived from the Gibbs free energy as follows (see Eq. (A7) in Ref. 23):

$$
\eta_{\mathrm{Li}}=\mu_{0}+g R T \frac{c_{\mathrm{Li}}}{c_{\max }}+R T \ln \frac{c_{\mathrm{Li}}}{c_{\max }-c_{\mathrm{Li}}}-\Omega_{\mathrm{Li}} \sigma_{h}+z_{\mathrm{Li}} F \phi,
$$

where $\sigma_{h}=(1 / 3) \boldsymbol{\sigma}: 1$ is the hydrostatic stress, $z_{\mathrm{Li}}=+1$ is the number of charges per one lithium ion, $\mu_{0}$ is the reference chemical potential in the system, $R$ is the ideal gas constant, $T$ is the absolute temperature, $c_{\max }$ is the stoichiometric maximum lithium concentration, and $g$ is a dimensionless parameter, characterizing the inter-ionic interactions described in Appendix B. ${ }^{23}$ We assume here that the temperature is constant in the whole system, therefore the chemical energy depends only on the concentration of lithium $c_{\mathrm{Li}}$.

The electrochemical potential of electrons is derived as well from the Gibbs free energy as follows (see Eq. (A8) in Ref. 23):

$$
\eta_{e}=z_{e} F \phi,
$$

where $F$ is the Faraday constant and $z_{e}=-1$ is the number of charges per electron.

From Eq. (4), the flux of lithium ions for single phase concentrations and electrons can be defined as

$$
\begin{gathered}
J_{\mathrm{Li}}=-c_{\mathrm{Li}} M_{\mathrm{Li}} \nabla \eta_{\mathrm{Li}} \\
=-c_{\mathrm{Li}} M_{\mathrm{Li}}\left\{\frac { 1 } { c _ { \mathrm { Li } } } R T \left(1+\frac{c_{\mathrm{Li}}}{c_{\text {max }}-c_{\mathrm{Li}}}+g \frac{c_{\mathrm{Li}}}{c_{\text {max }}}\right.\right. \\
\left.\left.+\frac{\mathrm{d} g}{\mathrm{~d} c_{\mathrm{Li}}} \frac{c_{\mathrm{Li}}^{2}}{c_{\max }}\right) \nabla c_{\mathrm{Li}}-\Omega_{\mathrm{Li}} \nabla \sigma_{h}+z_{\mathrm{Li}} F \nabla \phi\right\}, \\
\boldsymbol{J}_{e}=-M_{e} \nabla \eta_{e}=-M_{e} z_{e} F \nabla \phi,
\end{gathered}
$$

with the mobility of lithium ions $M_{\mathrm{Li}}$ and the mobility of electrons $M_{e}$,

$$
\begin{gathered}
M_{\mathrm{Li}}=\frac{D_{0}}{R T}\left(1-\frac{c_{\mathrm{Li}}}{c_{\text {max }}}\right), \\
M_{e}=\frac{\kappa_{e}}{z_{e}^{2} F^{2}},
\end{gathered}
$$

where $D_{0}$ is the diffusion coefficient of $\mathrm{Li}$ and $\kappa_{e}$ is the electric conductivity of $\mathrm{LiMn}_{2} \mathrm{O}_{4}$. Solid solutions of the two phases are introduced later in Eq. (10) and thoroughly detailed in Ref. 19 (see Appendix B in Ref. 23). The definition implies that the mobility of lithium ions depends on the lithium concentration, and that the lithium mobility tends to zero when its concentration reaches the maximum $c_{\max }$. A hypothesis from these two flux definitions is that there is no coupling between electrons and ions, where the electrochemical potential of one charge carrier influences the flux of the other one. ${ }^{25}$ However, it was shown that electron conduction in this spinel is mediated by hopping of small non-adiabatic polarons. ${ }^{1,26-28}$ It was theoretically shown that the polaron hopping can impact lithium ion movements ${ }^{28}$ and experimentally deduced that lithium diffusivity is intrinsically related to the polaronic properties. ${ }^{29}$ As this coupling is little understood and it is hard to quantify, it will be reserved to future studies.

Replacing the mobility of lithium ions from Eq. (8) to Eq. (6) yields

$$
\begin{aligned}
J_{\mathrm{Li}}= & -D_{0} D_{\mathrm{eff}} \nabla c_{\mathrm{Li}}+\frac{D_{0} \Omega_{\mathrm{Li}} c_{\mathrm{Li}}}{R T}\left(1-\frac{c_{\mathrm{Li}}}{c_{\max }}\right) \nabla \sigma_{h} \\
& -\frac{D_{0} z_{\mathrm{Li}} F c_{\mathrm{Li}}}{R T}\left(1-\frac{c_{\mathrm{Li}}}{c_{\max }}\right) \nabla \phi,
\end{aligned}
$$

which depends on the lithium concentration and its gradient $\nabla c_{\mathrm{Li}}$, hydrostatic stress $\nabla \sigma_{h}$, and electric potential $\nabla \phi . D_{\text {eff }}$ is the normalized effective diffusivity that translates the irregularity in the Gibbs free energy. It impacts the diffusivity and depends on $g$ (see Appendix B in Ref. 23). Multiplying $D_{\text {eff }}$ by $D_{0}$ yields the total effective diffusion coefficient. It has been already derived for $\mathrm{LiMn}_{2} \mathrm{O}_{4}$ by $\mathrm{Bohn}^{30}$ and is defined as a piecewise function for the range $0<c_{\mathrm{Li}}<c_{\max }$. It is plotted in Fig. 1 and represents both single phase and solid solutions of two phases.

Two models were implemented in the current work:

simple model, where the diffusivity is kept constant $\left(D_{\text {eff }}=1\right)$ as it is the case in previous models of ESM.

extended model, where the effective diffusivity $D_{\text {eff }} D_{0}$ is used $\left(D_{\text {eff }}=\right.$ Fig. 1$)$. Here, the phase transition is taken into account.

The reason for exploiting both models is given in Section IV C.

Based on the Nernst-Einstein relation, the electric current density $I$ is defined as

$$
\begin{aligned}
\boldsymbol{I}= & \boldsymbol{I}_{e}+\boldsymbol{I}_{\mathrm{Li}}=z_{e} F \boldsymbol{J}_{e}+z_{\mathrm{Li}} F \boldsymbol{J}_{\mathrm{Li}}=-\kappa_{e} \nabla \phi+z_{\mathrm{Li}} F \boldsymbol{J}_{\mathrm{Li}} \\
= & -D_{0} D_{\mathrm{eff}} z_{\mathrm{Li}} F \nabla c_{\mathrm{Li}}+\frac{D_{0} z_{\mathrm{Li}} F \Omega_{\mathrm{Li}} c_{\mathrm{Li}}}{R T}\left(1-\frac{c_{\mathrm{Li}}}{c_{\text {max }}}\right) \nabla \sigma_{h} \\
& -\left\{\kappa_{e}+\frac{D_{0} z_{\mathrm{Li}}^{2} F^{2} c_{\mathrm{Li}}}{R T}\left(1-\frac{c_{\mathrm{Li}}}{c_{\text {max }}}\right)\right\} \nabla \phi .
\end{aligned}
$$




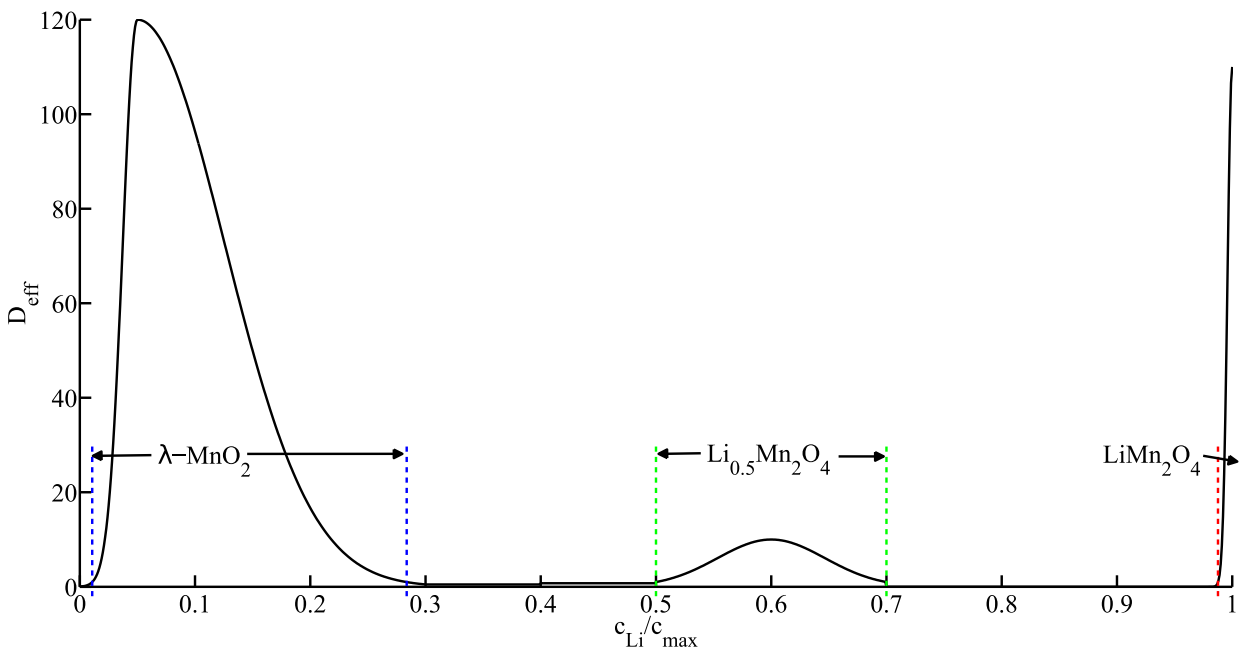

FIG. 1. $D_{\text {eff }}$ as function of $c_{\mathrm{Li}} / c_{\max }$. The three phases of spinel $\mathrm{Li}_{x} \mathrm{Mn}_{2} \mathrm{O}_{4}$ are marked on the plot. Colored bars are used in Fig. 7. Reproduced with permission from E. Bohn, "Partikel Modell fur Lithium Diffusion und mechanische Spannungen einer Interkalationselektrode," Ph.D. thesis (Karlsruher Institut fur Technologie, 2011). Copyright 2011 Shaker Verlag $\mathrm{GmbH}$.

In order to model the system, we need to use balance laws, such as balance of momentum for the mechanical stress

$$
\nabla \cdot \boldsymbol{\sigma}=\mathbf{0},
$$

the conservation of mass

$$
\frac{\partial c_{\mathrm{Li}}}{\partial t}+\nabla \cdot \boldsymbol{J}_{\mathrm{Li}}=0
$$

and the conservation of charge

$$
\frac{\partial \rho}{\partial t}+\nabla \cdot \boldsymbol{I}=0
$$

The $\mathrm{LiMn}_{2} \mathrm{O}_{4}$ particle is connected to a closed circuit, thus the electron influx and electron outflux are the same. Following García et al., ${ }^{31}$ we assume that there is no local charge accumulation $(\partial \rho / \partial t=0)$, so that Eq. (14) simplifies to

$$
\nabla \cdot \boldsymbol{I}=0
$$

As polaron/ion coupling is an important and poorly known mechanism, ${ }^{1,26-29}$ local charge accumulation seemed to be not significant enough to be implemented, but this actually remains an open question.

\section{B. Mean electric force}

Section III A has dealt with the model describing lithium ion reorganization within $\mathrm{LiMn}_{2} \mathrm{O}_{4}$ during and after a DC pulse (time domain). In this section, we outline how the ESM signal is estimated from the output of the model. The $\mathrm{AC}$ excitation (frequency domain) is not directly modeled but only suggested through a mean electric force.

As explained Sec. I, we consider that the ESM signal does not originate from diffusion of lithium as the excitation frequency is too high for allowing hopping significant enough to be detected through Vegard's deformation. ${ }^{11}$ Instead, we consider the signal to be driven by ionic polarization mechanisms, where the lithium ions are vibrating within their interstitial sites without long-range hopping. The ionic vibration is induced by the electric force and in return induces the ESM signal. An alternative electric potential $\phi_{A C}(t)$ of frequency $\omega$ is applied without interruption during experiment. Here, the electric potential is given as follows:

$$
\phi_{A C}(t)=\sqrt{ } 2 \phi_{A C}^{r m s} \cos (\omega t),
$$

where $\phi_{A C}^{r m s}$ is the root mean square (RMS) voltage. Considering quasi-static motions, the magnetic field produced by the alternating electric field is ignored. ${ }^{32}$ The mean electric force $\mathfrak{F}_{A C}(t)$ applied onto the ions is

$$
\mathfrak{F}_{A C}(t)=\int_{V} z_{\mathrm{Li}} c_{\mathrm{Li}}(t) F \boldsymbol{E} \mathrm{d} V,
$$

where $V$ is the volume of the body and $\boldsymbol{E}=-\nabla \phi_{A C}$ is the mean electric field, with $\phi_{A C}$ the local RMS electric potential. $\phi_{A C}$ is equal to $\phi_{A C}^{r m s}$ at the tip contact and is calculated by COMSOL in the rest of the body. It is constant in time, consequently $\boldsymbol{E}$ is also constant in time and $\mathfrak{F}_{A C}$ varies in time only with the concentration field $\left(c_{\mathrm{Li}}(t)\right)$. This force is the static part of Lorentz force. The ESM signal $S_{\text {Total }}(t)$ measured after the release of the DC voltage is considered to be the sum of a constant background noise $S_{\text {background }}$ and a time-dependent signal originating from the lithium ions $S_{\mathrm{Li}}(t)$. In a first approximation, the latter is considered linearly proportional to $\mathfrak{F}_{A C}(t)$,

$$
S_{\mathrm{Li}}(t) \propto \mathfrak{F}_{A C}(t)
$$

Since the real underlying mechanism is unknown, $\mathfrak{F}_{A C}$ should be understood as a mathematical tool to probe the concentration of lithium just under the tip. Equation (17) is in fact a weighted sum of $c_{\mathrm{Li}}$, where the weight is given by the amplitude of the electric field $\boldsymbol{E} . S_{\mathrm{Li}}(t)$ probes consequently $c_{\mathrm{Li}}$ within a small volume.

\section{Boundary conditions and implementation}

The modeled body consists of a spinel $\mathrm{Li}_{x} \mathrm{Mn}_{2} \mathrm{O}_{4}$ single crystal particle with an initial arbitrary normalized concentration of lithium ions $\hat{c}_{i n i}=c_{0} / c_{\max }$. It is a half-spherical particle of radius $R_{\text {part }}$. A top view of the particle with the mechanical boundaries is schematically shown in Fig. 2(a). These boundaries do not allow rigid body motion but there is 


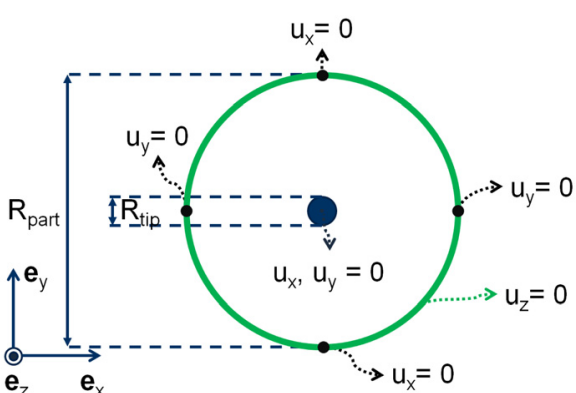

(a)

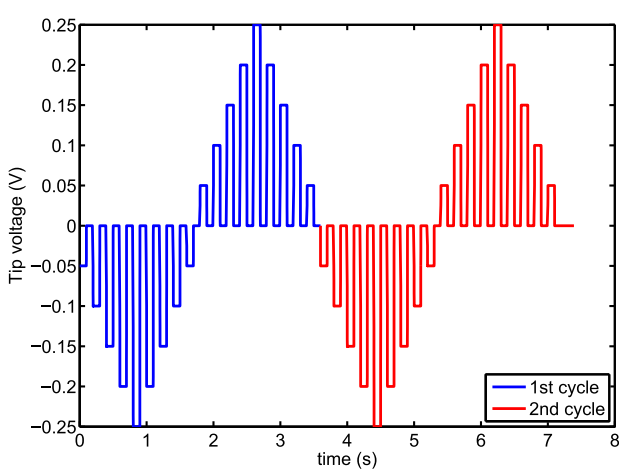

(b)
FIG. 2. (a) Displacement boundary condition. Conditions on displace ments are indicated by the letter $u$ with the direction in subscript. (b) Voltage steps used in the simulation of voltage spectroscopy measurements. no counter mechanical force on the particle, hence it is free to swell. Lithium ions can freely move within the particle if enough vacancies are available $\left(c_{\mathrm{Li}}<c_{\max }\right)$. There is no flux of lithium across the boundaries, i.e., the total amount of lithium ions stays the same within the particle

$$
\begin{aligned}
\frac{\int_{V} \hat{c} \mathrm{~d} V}{V} & =\hat{c}_{i n i}, \\
\boldsymbol{J}_{\mathrm{Li}} \cdot \boldsymbol{n} & =0,
\end{aligned}
$$

where $\hat{c}=c_{\mathrm{Li}} / c_{\max }$ is the normalized lithium concentration and $\boldsymbol{n}$ is the surface unit vector. The electric potential applied by the AFM tip $\phi_{D C}$ is defined as

$$
\phi_{D C}=\phi_{0}(t) \frac{R_{\text {tip }}^{2}}{\left(\left(x-x_{0}\right)^{2}+\left(y-y_{0}\right)^{2}+R_{\text {tip }}^{2}\right)},
$$

where $\phi_{0}(t)$ is the time dependent applied voltage and $R_{\text {tip }}$ is the tip radius centered at position $\left(x_{0}, y_{0}\right) . \phi_{D C}$ is applied over the whole top flat surface but is locally distributed within a circle of radius $R_{\text {tip }}$ with smooth edges following a 2D Lorentz-like function (see Ref. 33). The remainder of the surface is grounded to mimic a perfect ohmic contact with carbon black. Note that the dimensions of the particle are big enough as compared to $R_{\text {tip }}$ so that most of the electric field is concentrated in the vicinity of the tip. Schematics are shown in Figure D2 of Appendix D in Ref. 23.

The model is implemented in COMSOL Multiphysics 4.4 with a segregated (or iterative) solver for time dependent problems, where two coefficients from partial differential equation modules are used for Eqs. (13) and (15). Equation (12) is put in the solid mechanics module. For modeling time-spectroscopy measurements as explained in Section II, $\phi_{0}(t)$ is described by a gate function where

$$
\phi_{0}(t)=\left\{\begin{array}{l}
\Phi_{0} \text { for } 0<t<10 \mathrm{~ms} \\
0 \text { otherwise }
\end{array}\right.
$$

where $\Phi_{0}$ is the applied voltage. The gate function is gradually increased over a period of $0.1 \mathrm{~ms}$. Concerning simulation of the voltage spectroscopy, the voltage is plotted versus time in Fig. 2(b) (pulse time of $100 \mathrm{~ms}$ smoothed over a period of $1 \mathrm{~ms}$ ).

In order to simulate the ESM signal, a supplementary electrostatics physics module was used with no coupling to any variable used in the other modules. It is merely aimed at describing the AC electric field $-\nabla \phi_{A C}$ within the body when the AC voltage $\phi_{A C}$ is applied. $\phi_{A C}^{r m s}$ is applied in the same way as $\phi_{D C}$ (see Eq. (19)).

The mesh consists of 10920 elements with a high refinement in the vicinity of the AFM tip (see Figure D3 in Ref. 23). Default parameters are listed in Table I.

\section{RESULTS AND DISCUSSION}

\section{A. Experiment}

\section{Voltage spectroscopy}

Figure 3 shows hysteresis loops taken on the samples obtained from the fresh and aged batteries with $0 \%$ SoC. Typical concentration-controlled loops are measured. ${ }^{35}$ Negative DC voltages applied to the tip attract mobile $\mathrm{Li}$ ions to the tip-sample contact point and increase the local $\mathrm{Li}$ concentration, thus increasing the ESM response. Positive DC voltages repulse $\mathrm{Li}$ ions and reduce local Li concentration, thereby decreasing the ESM response. Ideal ESM response is attributed to surface vibrations caused by lithium ions. However, the lock-in detection system of the microscope can measure other AC-mediated responses as

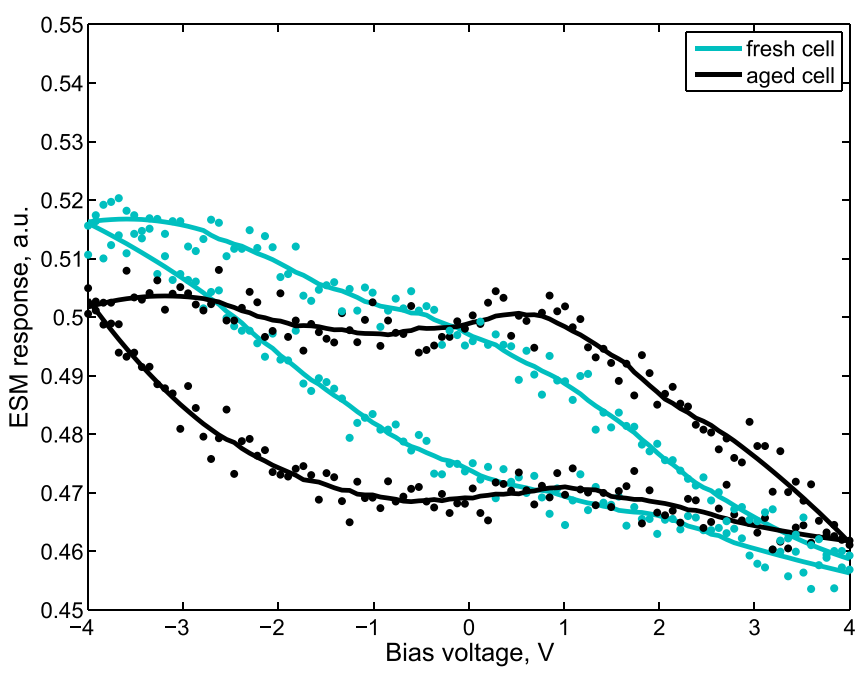

FIG. 3. Experimental amplitude hysteresis loops measured on fresh $\mathrm{LiMn}_{2} \mathrm{O}_{4}$ particles (green curves) and aged $\mathrm{LiMn}_{2} \mathrm{O}_{4}$ particles (black curves). The dots represent the average of three loops, the solid lines are smoothed versions. 
TABLE I. Default parameters.

\begin{tabular}{lccc}
\hline \hline Property & Symbol & Value & Unit \\
\hline Electrical conductivity & $\kappa_{e}$ & $10^{-2}$ & $\mathrm{~S} \mathrm{~m}^{-1}$ \\
Lithium diffusion & $D_{0}$ & $10^{-14}$ & $\mathrm{~m}^{2} \mathrm{~s}^{-1}$ \\
Molar volume expansion & $\Omega_{\mathrm{Li}}$ & $3.5 \times 10^{-6}$ & $\mathrm{~m}^{3} \mathrm{~mol}^{-1}$ \\
Maximum concentration & $c_{\text {max }}$ & 22900 & $\mathrm{~mol} \mathrm{~m}^{-3}$ \\
Initial concentration & $c_{\text {ini }}$ & 0.5 & $\ldots$ \\
Elastic modulus & $E$ & 100 (Refs. 21 and 34) & $\mathrm{GPa}$ \\
Poisson's ratio & $\nu$ & 0.3 & $\ldots$ \\
Temperature & $\mathrm{T}$ & 293.15 & $\mathrm{~K}$ \\
Tip radius & $R_{\text {tip }}$ & 0.05 & $\mu \mathrm{m}$ \\
Particle radius & $R_{\text {part }}$ & 10 & $\mu \mathrm{m}$ \\
Applied voltage & $\bar{\phi}_{0}$ & 0.1 & $\mathrm{~V}$ \\
\hline \hline
\end{tabular}

explained in Section I. Taking into account that $\mathrm{LiMn}_{2} \mathrm{O}_{4}$ is a small polaron semiconductor, ${ }^{27}$ an additional contribution can emerge from AC mediated dipoles. Chen et al. ${ }^{35}$ suggested that voltage spectroscopy hysteresis loops can be used to distinguish between polar and non-polar contributions. Polar contribution would give rise to butterfly-like amplitude loops, while concentration-dependent contributions would result in simple hysteresis loops. The loops in Fig. 3 refer to the most common measurement on our samples but butterfly-like loops were also obtained (see Fig. C1 in Ref. 23 ). It can be noticed that the fresh sample produces a more symmetrical loop than the aged sample, for which the loop is also more opened.

ESM loops are a too complex type of measurement to extract qualitative data with a clear physical meaning. Each data point depends on the length of the pulse, the history of the measurement (the accumulation of all the previous pulses in the spectroscopy), and the time lap between the release of the DC pulse and the data point (longer time laps give narrower loops). Voltage spectroscopy has the advantage of giving a qualitative overview within seconds of voltage dependencies in the ESM signal and the reversibility of the measurement. Time spectroscopy is a more appropriate measurement to quantitatively study diffusion processes.

It is important to note that voltage spectroscopy was also carried out on pure epoxy and the aluminum current collector and they exhibited no loops.

\section{Time spectroscopy}

Figure 4 shows typical relaxation of the ESM response after DC pulses of different magnitudes on the same location. A higher DC voltage pulse results in larger change of local $\mathrm{Li}$ concentration below the tip and, hence, in higher initial ESM response. Relaxation time of about $1 \mathrm{~s}$ is in agreement with the diffusion relaxation time estimated in Section I. It is much slower than the space charge relaxation time. Figure 5 shows two of the same measurements after normalization (gray and black lines). They were normalized by first subtracting the average signal before the DC pulse (background) and then by dividing by the first value when DC is off (extremum). They show the same relaxation time. Besides, the same measurements were carried out on the other fresh samples opened at 50\% and 100\% SoC. They also show the same speed of relaxation even though different diffusion coefficients are expected for different lithium concentrations as experimentally measured by macroscopic titration techniques ${ }^{36,37}$ and as described by Figure 1. Experimental measurements show a non-linear change within a range of one order of magnitude, ${ }^{36,37}$ hence it is possible that these three samples with different SoCs exhibit similar diffusion coefficient. Additionally, the time spectroscopy signal has more complex underlying mechanisms than titration techniques due to the significant concentration change below the tip, where the whole range of diffusion parameters could act in parallel. Per contra, time spectroscopy done on the aged sample produces a much slower relaxation to the initial state. Being fitted with an appropriate physical model, the relaxation of the normalized ESM response can be thus used to estimate the local Li diffusion coefficient.

Note that similar measurements with longer, $100 \mathrm{~ms}, 10 \mathrm{~V}$ DC pulses induced irreversible processes (irreproducible measurements). Irreversible processes, such as Li extraction and its reaction with absorbed water and atmosphere gases, could emerge and contribute to the response as well when the applied DC pulse is higher or longer than a certain threshold value (in voltage or time). These contributions are difficult to assess numerically because they depend on the sample properties and experimental conditions (humidity, temperature, gases, etc.). Nevertheless, they lead to irreversible changes of the

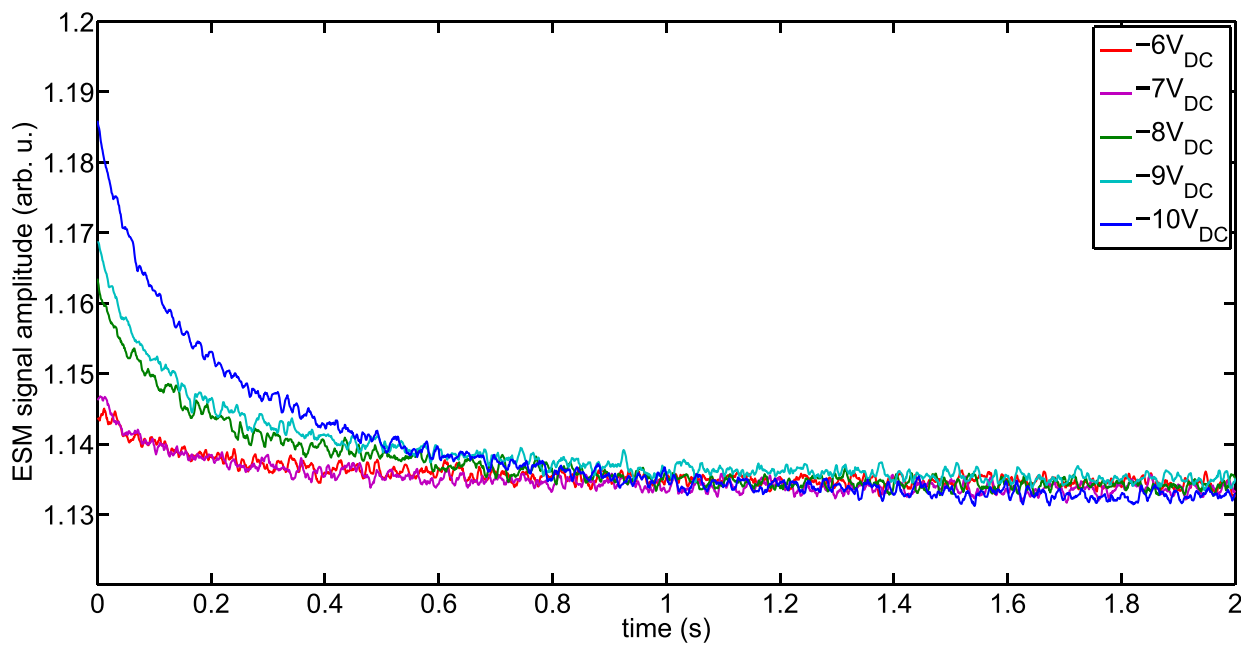

FIG. 4. Relaxation curves after applica tion of rectangular $10 \mathrm{~ms}$ DC pulses of $6,7,8,9$, and $10 \mathrm{~V}$ to the tip. Each curve represents an average of five reproducible consecutive measurements. 


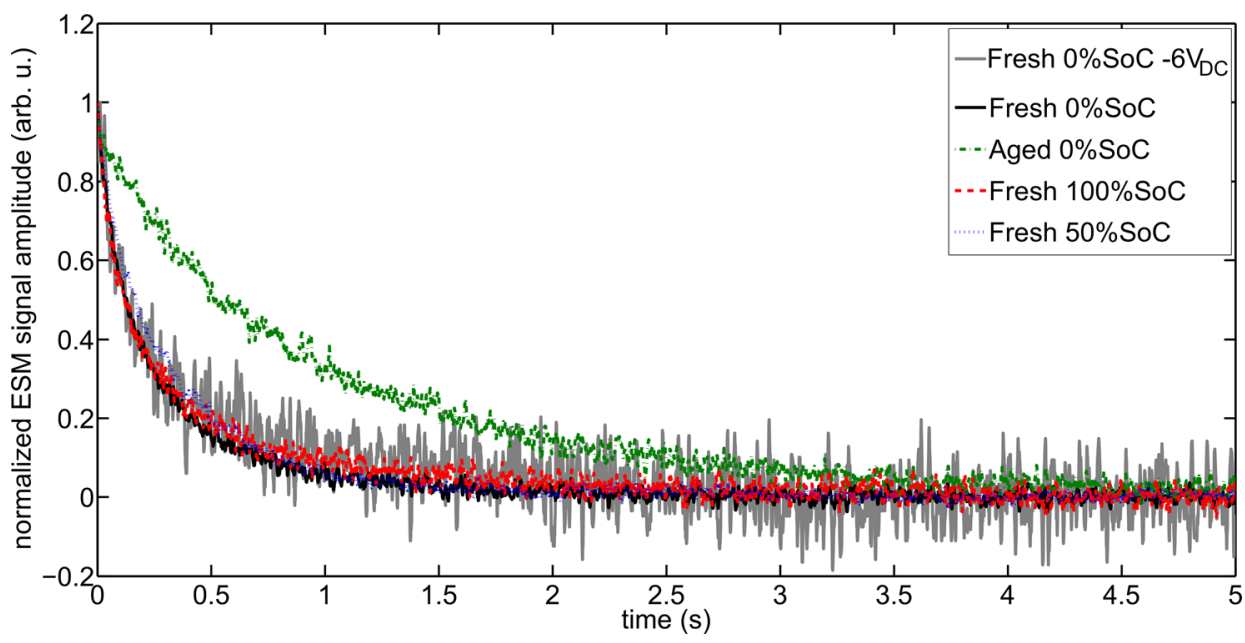

FIG. 5. Normalized relaxation curves after application of rectangular $10 \mathrm{~ms}$ DC pulses of $10 \mathrm{~V}$ (unless otherwise stated) to the tip on three fresh samples of different states of charge $(\mathrm{SoC})$ and one aged sample of $0 \%$ SoC.

topography and thus can be detected. Similar measurements were also done on epoxy and with the AFM tip lifted. Relaxation response was not detected for any applied DC voltages (up to $10 \mathrm{~V}$ ).

\section{B. Model}

\section{Voltage spectroscopy}

Two loops per model were calculated by producing a data point with the first value of $\mathfrak{\varpi}_{A C}$ after each voltage drop with the default parameters from Table I. They can be seen in Fig. 6. It can be noticed how the simple model (Figure 6(a)) yields a relatively symmetrical hysteresis loop, while the other one (extended model) is flattened towards the negative potentials (Figure 6(b)). As $\mathfrak{F}_{A C}$ is directly connected to the local concentration of ions just below the tip, there is a direct relation to the dependence depicted in Fig. 1, in which $\mathrm{LiMn}_{2} \mathrm{O}_{4}$ would avoid local concentration associated with $D_{\text {eff }} \ll 1$, i.e., for concentrations between 0.7 and 0.98 . This phenomenon is illustrated in Fig. 7. When the positive pulses

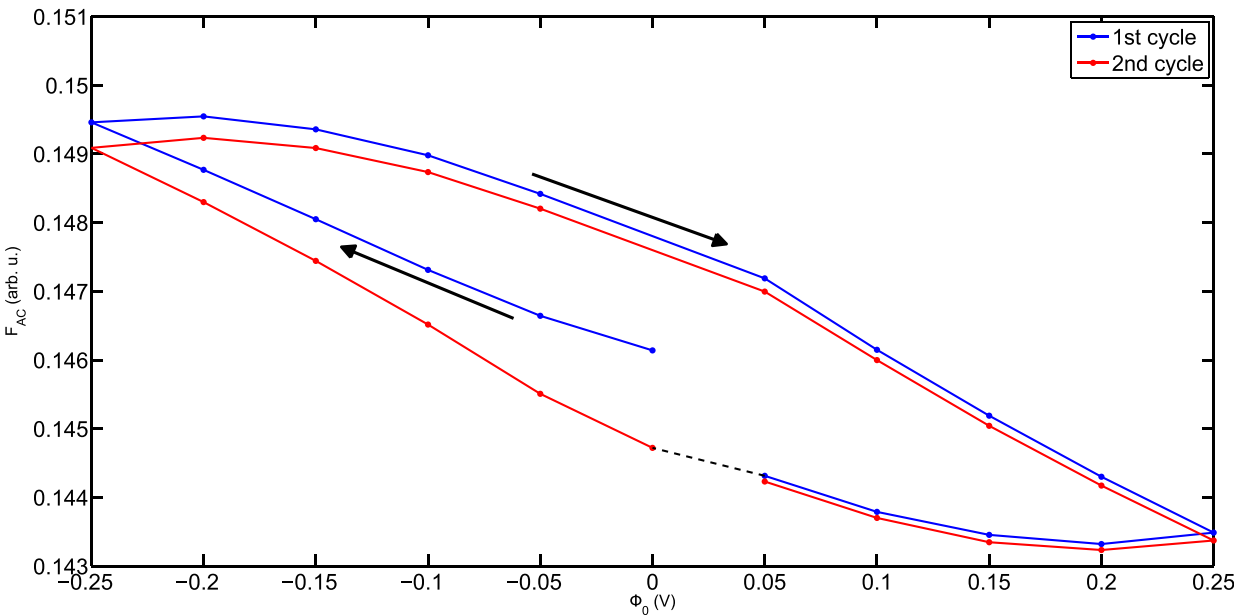

(a)

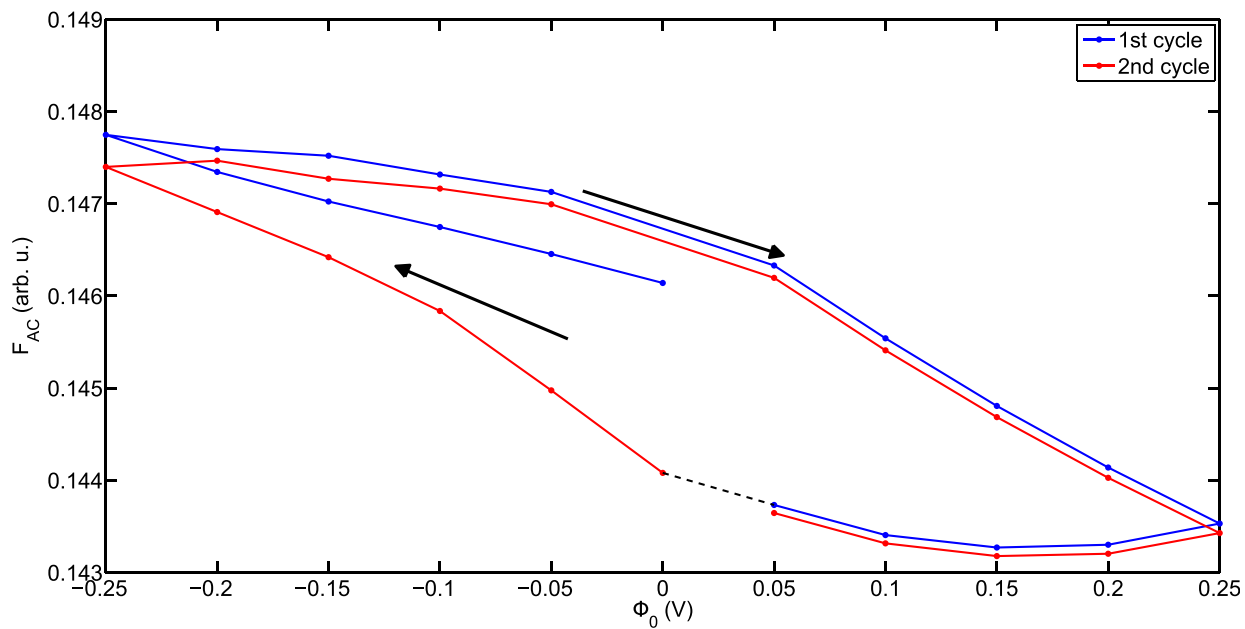

(b)
FIG. 6. Simulated ESM hysteresis loops for the two models: (a) simple model ( $\left.D_{\text {eff }} 1\right)$ and (b) extended model ( $D_{\text {eff }}$ from Figure 1). Data points are constructed by associating each voltage pulse with the first $\mathfrak{F}_{A C}$ value obtained when DC returns to 0 . 


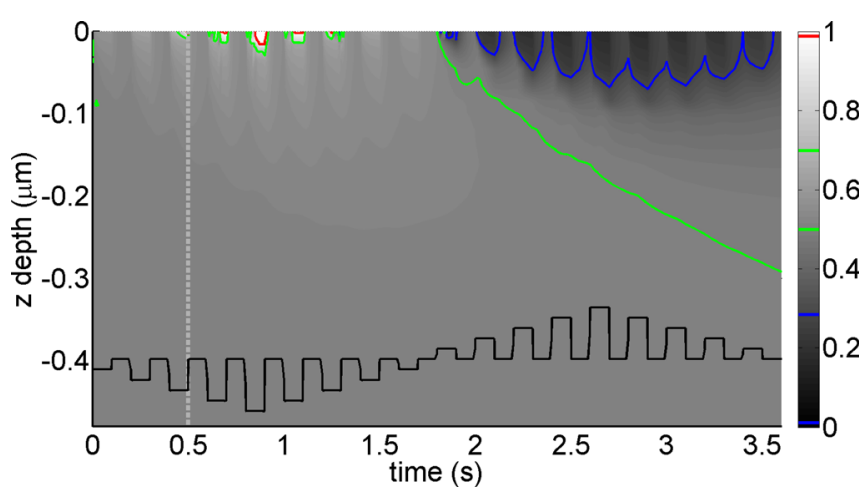

FIG. 7. Evolution of the concentration of lithium ions below the tip down to a depth of $0.5 \mu \mathrm{m}$ during the first loop taking into account $D_{\text {eff }}$. The bright ness is correlated with the concentration of lithium $c_{\mathrm{Li}}$ as indicated by the color bar. Colored contours represent each phase of spinel $\mathrm{Li}_{x} \mathrm{Mn}_{2} \mathrm{O}_{4}$, with blue for poorly lithiated spinel, green for the middle phase, and red for lithi ated spinel as marked in Fig. 1. These boundaries are located where $D_{\text {eff }}\left(c_{\mathrm{Li}}\right) \sim 1$. The black line represents the applied voltage versus time. The same plot for the simple model is added in Appendix D in Ref. 23. The gray dashed line indicates the position of data plotted in Fig. 8.

are applied (right hand side of the plot), the local concentration would jump from $c_{i n i}=0.5$ to the low concentration phase below 0.3 as the diffusivity tends to 0 between these two values. However, the system would not leave the 0.50 .7 concentration range for the negative pulses (left hand side) easily, explaining why the loops do not extend to higher $\mathfrak{F}_{A C}$ values. In the simple case (see Figure 8), it can be seen how the concentration regularly increases when approaching the surface while there is an abrupt and thin boundary between the two phases $\mathrm{Li}_{0.5} \mathrm{Mn}_{2} \mathrm{O}_{4}$ and $\mathrm{LiMn}_{2} \mathrm{O}_{4}$ with the extended model. It can be also seen how the system would slowly delithiate in the simple case while it would immediately come back to 0.5 with the extended model, trying to minimize the presence of this phase boundary. These different processes induced by phase transitions could explain the apparent asymmetry in the experimental loops of Figure 3.

\section{Time spectroscopy}

Figure 9 shows the evolution of $\mathfrak{F}_{A C}$ using the extended model for an initial concentration of 0.7 . This value was picked as it is an extreme case just at the boundary between the two phases. When applying a positive voltage pulse, the concentration below the tip first reduces and then slowly returns to its initial state (red dot). For a negative voltage pulse, the concentration below the tip increases and a small volume experiences an irreversible phase transition to $\mathrm{LiMn}_{2} \mathrm{O}_{4}$ and does not return to the initial concentration, hence the measured $\mathfrak{F}_{A C}$ converges to a higher value than the initial one (red dot). This effect can be reversed by applying a pulse of opposite sign as was shown experimentally in a previous report. ${ }^{14}$ The experimental plot from this reference is added to Appendix D in Ref. 23 (see Figure D5). Additionally, animations of the particle deformation using the extended model can be found in Appendix D in Ref. 23.

A parametric study was done only using the simple simulation model ( $g=0$ or $D_{\text {eff }}=1$ ) in order to obtain simplistic time dependent responses that can be equated.

Normalized time spectroscopy ESM signal and normalized $\hat{\mathfrak{F}}_{A C}$ for different applied voltages are plotted in Fig. 10. Both the experimental measurements and the simulation outputs are independent of the applied voltage once normalized. $\hat{\mathfrak{F}}_{A C}$ was calculated by setting the maximum value of $\mathfrak{F}_{A C}$ to 1 and the value before applying the DC pulse to 0 . If not normalized, a higher applied voltage would induce a higher $\mathfrak{F}_{A C}$ as for the experimental results plotted in Fig. 4.

Normalized time spectroscopy responses for different $D_{0}$ can be seen in Figure 11(a). They reproduce a typical relaxation process similar to that in real measurements (black dotted line), where the speed at which it returns to equilibrium depends on $D_{0}$.

If divided by their own time derivative (Figure 11(b)), they present an affine response. This behavior starts deviating for low $D_{0}$ and low $t$. Such behavior can be described by power laws of the form

$$
\left\{\begin{array}{l}
\frac{\hat{\mathfrak{F}}_{A C}(t)}{\mathrm{d} \hat{\mathfrak{F}}_{A C}(t) / \mathrm{d} t}=\left(p t+p^{\prime}\right) \\
\hat{\mathfrak{F}}_{A C}(0)=1
\end{array} \Longleftrightarrow \hat{\mathfrak{F}}_{A C}(t)=(a t+1)^{1 / p},\right.
$$

where $p$ is the negative first-order coefficient of the affine function and $a=p / p^{\prime}$ is positive. The curve described by a power law is made of two parts. The initial decay is controlled by $a / p$, which describes at which speed the signal

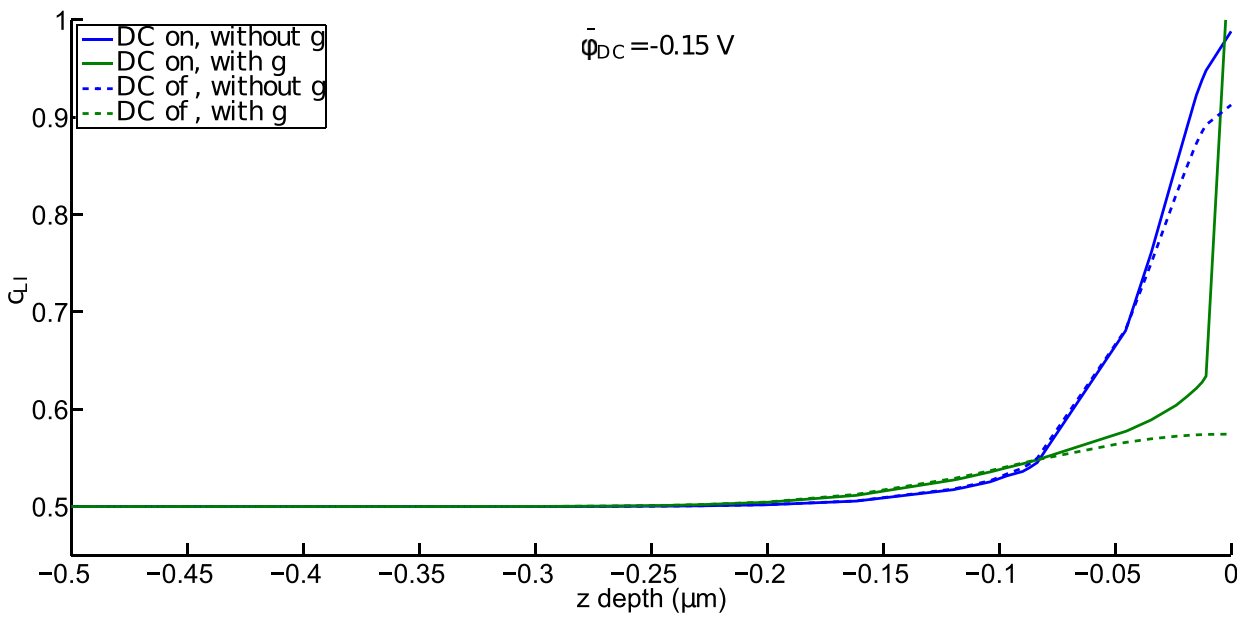

FIG. 8. Same information as in Fig. 7 with (green curves) and without (blue curves) using the extended model for $V_{D C} \quad 0.15 \mathrm{~V}$ just before switching the DC voltage off (solid curves) and after switching it off (dashed curves). 


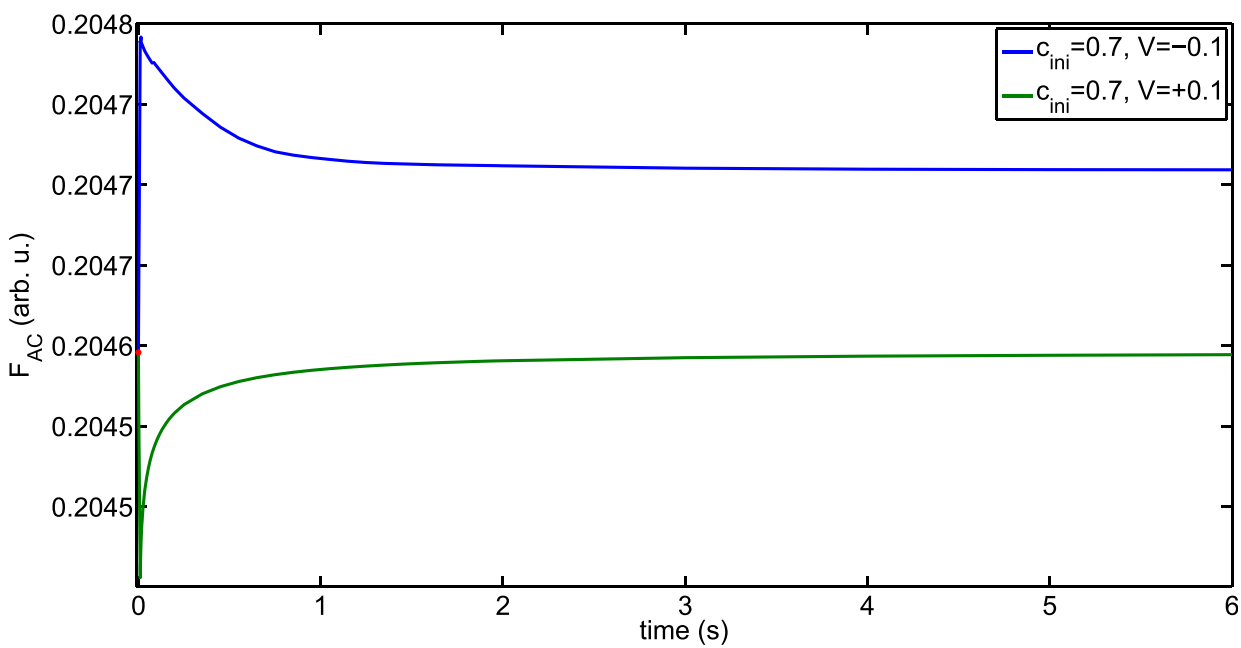

FIG. 9. Time Spectroscopy modeled on a particle with an initial concentra tion of 0.7. A positive bias produces a reversible signal (green curve), while a negative bias produces an irreversible signal (blue curve).

drops from 1 . The second part of the curve is controlled by $p$ and describes how low is the shoulder.

A parametric study was done, where one parameter would be varied, while the other ones would be equal to the default values (see Table I). The results are plotted in Figs. 12 and 13. $p$ only slightly depends on the parameters and is around -1 as shown in Fig. 12. It is strongly correlated with the tip radius. The parametric study for $a$ is plotted in Fig. 13. $a$ seems to be correlated with $D_{0}$ and $R_{\text {tip. The }}$ other parameters seem to have a limited impact for the ranges of the study. Even the initial concentration has a limited impact on the normalized signal. The thickness study would be done by modeling a half oblate spheroid particle of equatorial radius $R_{\text {part }}$ and of different short radii (or thicknesses). The behavior strongly deviates from the one described in Eq. (21) for thicknesses approaching the order of magnitude of $R_{\text {tip. }}$. For all other cases, the adjusted $\mathrm{R}$-square is above 0.99 when fitted with Eq. (21).

The power law resembles the analytical solution found by Morozovska et al. ${ }^{3}$ from Eq. (2). The exponent is not always equal to $-3 / 2(p=-2 / 3)$ but is somewhat close

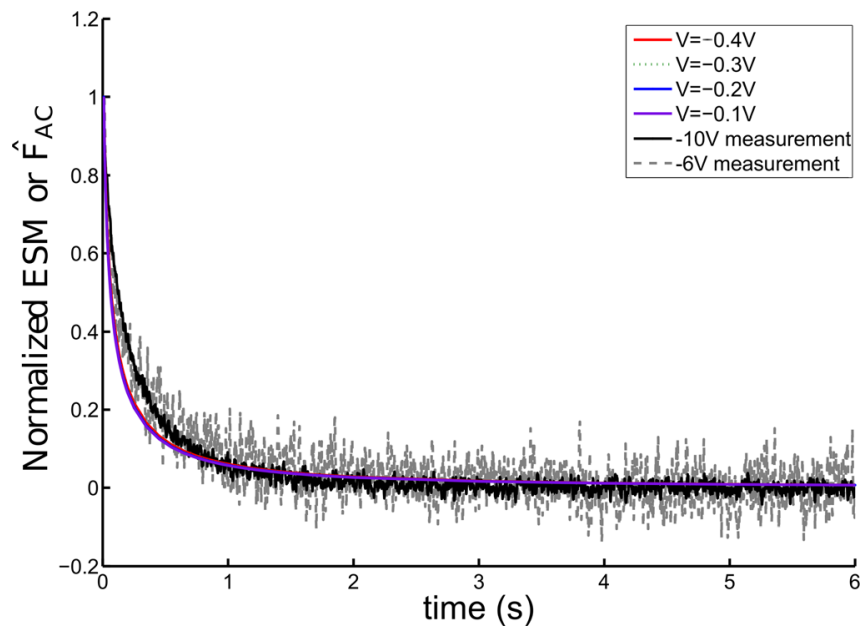

FIG. 10. Normalized ESM signal after a $10 \mathrm{~ms}, 10 \mathrm{~V}$ DC pulse (solid black line) and after a $10 \mathrm{~ms}, 6 \mathrm{~V} \mathrm{DC}$ pulse (dotted gray line). Normalized $\mathfrak{F}_{A C}$ from time spectroscopy simulations using the default parameters and differ ent applied voltages $\bar{\phi}_{0}$ (solid colored lines, voltages indicated in the legend). Normalization procedure explained in the text.
( $p=[-0.8,-1.4])$. The parameter $a$ had the following proportionality with $R_{\text {tip }}$ and $D_{0}$ :

$$
a \propto \frac{D_{0}^{1 / 2}}{R_{\mathrm{tip}}} .
$$

The same power law was found for the tip displacement $u_{z}(t)$ in our case with differences in the exponents of Eq. (22). As $u_{z}(t)$ is not a measurable quantity, further investigation on this matter is not published here.

\section{Equation fitting}

Fitting Eq. (21) to experimental measurements from Figure 5 yields the fitting coefficients shown in Table II. The first striking observation is that the exponent $p$ seems off the range previously computed even though the $95 \%$ confidence bounds provided by Matlab curve fitting toolbox are good (within $5 \%$ of the fitted values). Based on Eq. (22), the coefficient $a$ of the aged sample is one order of magnitude smaller than the other ones, which could be interpreted as a diffusion coefficient which is two orders of magnitude smaller (for all other parameters fixed), if the hypothesis of Eq. (18) stands. It is nevertheless not sufficient to obtain quantitative information without more rigorous description of Eq. (22) and without knowing the value of the other parameters in advance.

Besides, the current model is relatively simplistic. A better experimental/model comparison could be obtained if polaron-ion coupling is better understood, phase descriptions of spinel $\mathrm{Li}_{x} \mathrm{Mn}_{2} \mathrm{O}_{4}$ are taken into account and if experimental conditions are better controlled, e.g., no humidity is present and the temperature is constant.

\section{Origin of the signal and outlook}

$\mathfrak{F}_{A C}$ is used here as a mathematical tool rather than a real physical quantity to probe the concentration of lithium ions within a small volume below the tip. While it is believed that the normalized ESM signal is mainly driven by the change of concentration of ions below the tip, the Vegard contribution cannot be the origin of the signal under AC excitation. Other electromechanical couplings should be 


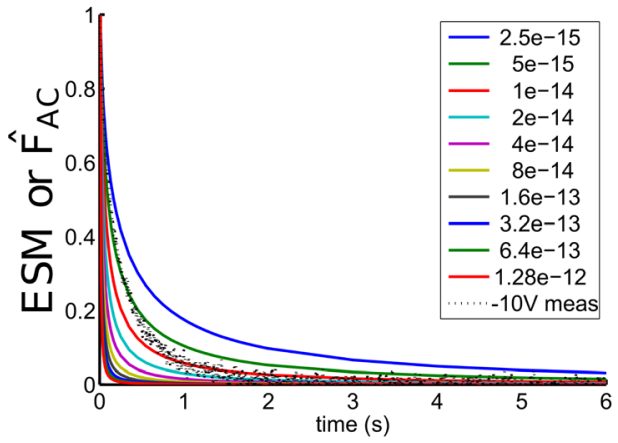

(a)

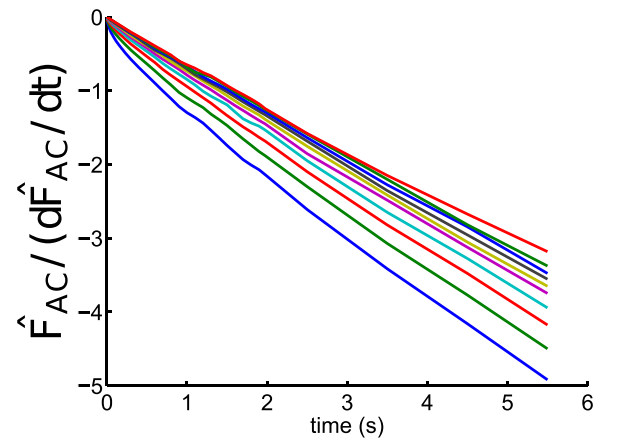

(b)
FIG. 11. (a) Normalized ESM signal after a $10 \mathrm{~ms}, 10 \mathrm{~V}$ DC pulse (dotted black line) in comparison with normal ized $\mathfrak{F}_{A C}$ simulated using the default parameters and different diffusion coefficients (solid colored lines, coeffi cient values in the legend). (b) $\mathfrak{F}_{A C}$ di vided by its time derivative (same legend). considered, including but not limited to converse flexoelectricity or electrostriction. Unlike $\mathfrak{F}_{A C}$ which is linearly dependent on $\boldsymbol{E}$, the former would be dependent on $\nabla \boldsymbol{E}$ and the latter on $\boldsymbol{E}^{2} \cdot{ }^{38,39}$ The butterfly-like loops could originate from the electrostrictive effect. ${ }^{35}$ It is possible that the same effect is present even in the case of diamond-like loops, hence the first and second harmonic responses should be measured in future studies (see Ref. 35). Other potential mechanisms are given in Appendix in Ref. 23.

With better knowledge of the underlying mechanisms, future work on ESM should focus on improving the definition of $S_{\mathrm{Li}}$ in Eq. (18). In any case, the coupling should increase for higher lithium concentration as seen experimentally (see, e.g., Fig. 4 or Ref. 9). The physical origin is not necessarily directly related to lithium concentration: it could depend on the valence of the transition metals, i.e., the local ratio of $\mathrm{Mn}^{3+}$ versus $\mathrm{Mn}^{4+}$, or it could be coupled to polarons.
It should be additionally noted that even if $S_{\mathrm{Li}}$ is fully understood, measuring $c_{0}$ (initial concentration) quantitatively by ESM would not be possible due to the omnipresent background signal $S_{\text {background }}$ which is a composite response depending on $c_{0}$ and other effects as discussed in Section I. Hence, ESM-TS can only probe the diffusivity, i.e., the speed of concentration change.

\section{CONCLUSION}

A model was developed to simulate electrochemical strain microscopy using the time spectroscopy mode. Previous analyses showed that an ESM signal driven only by Vegard deformation would lead to unobservable signals even though experimental observations exist. In this work, we suggest to associate the ESM signal with the concentration of lithium within a small volume under the tip as described by the mean AC electric field (see Eq. (17)). Besides, a concentration
' $p$ ' in $\left(a^{*} t+1\right)^{1 / p}$
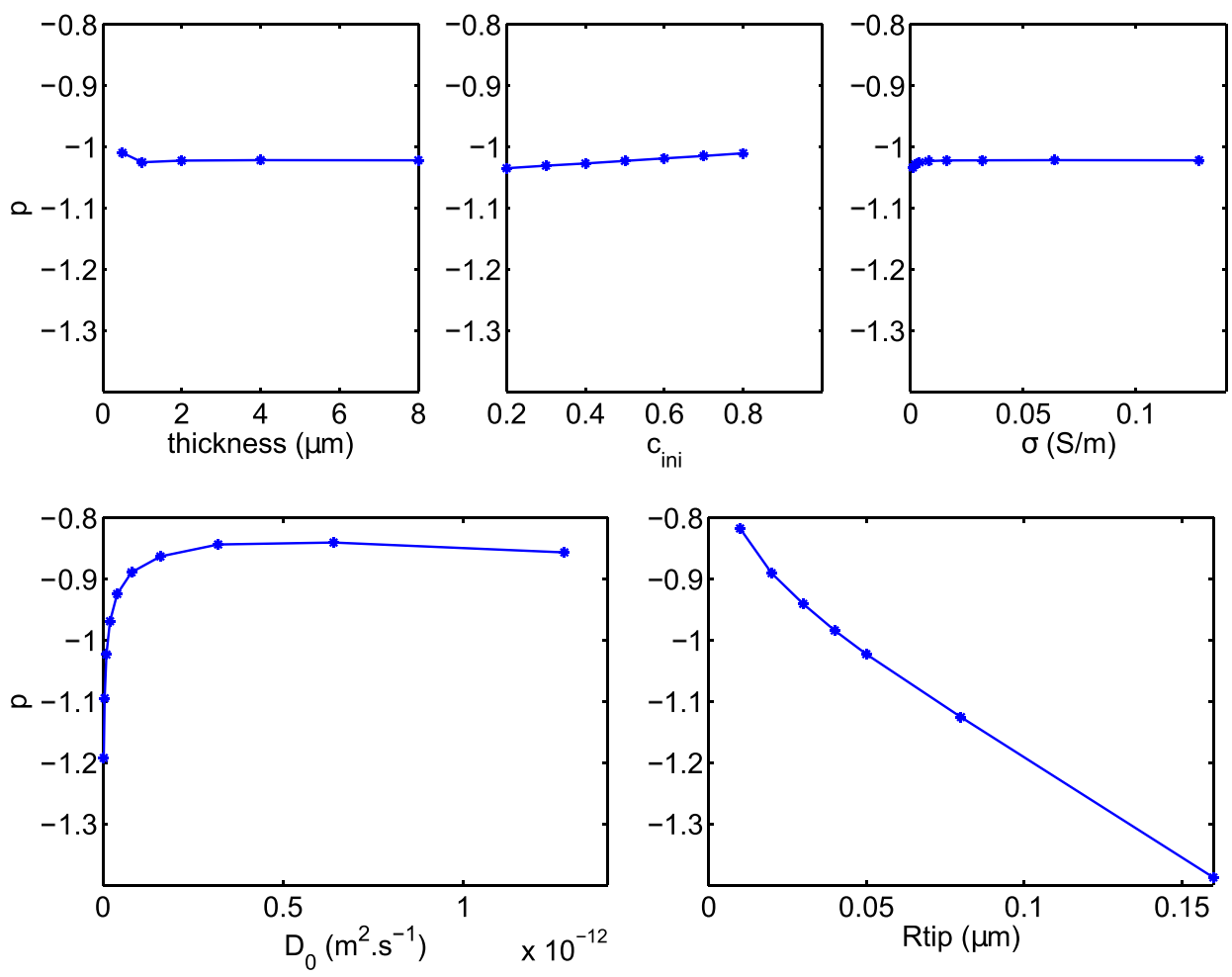

FIG. 12. Parameter study for $p$ with respect to the default state. 


$$
\text { 'a' in }\left(a^{*} t+1\right)^{1 / p}
$$
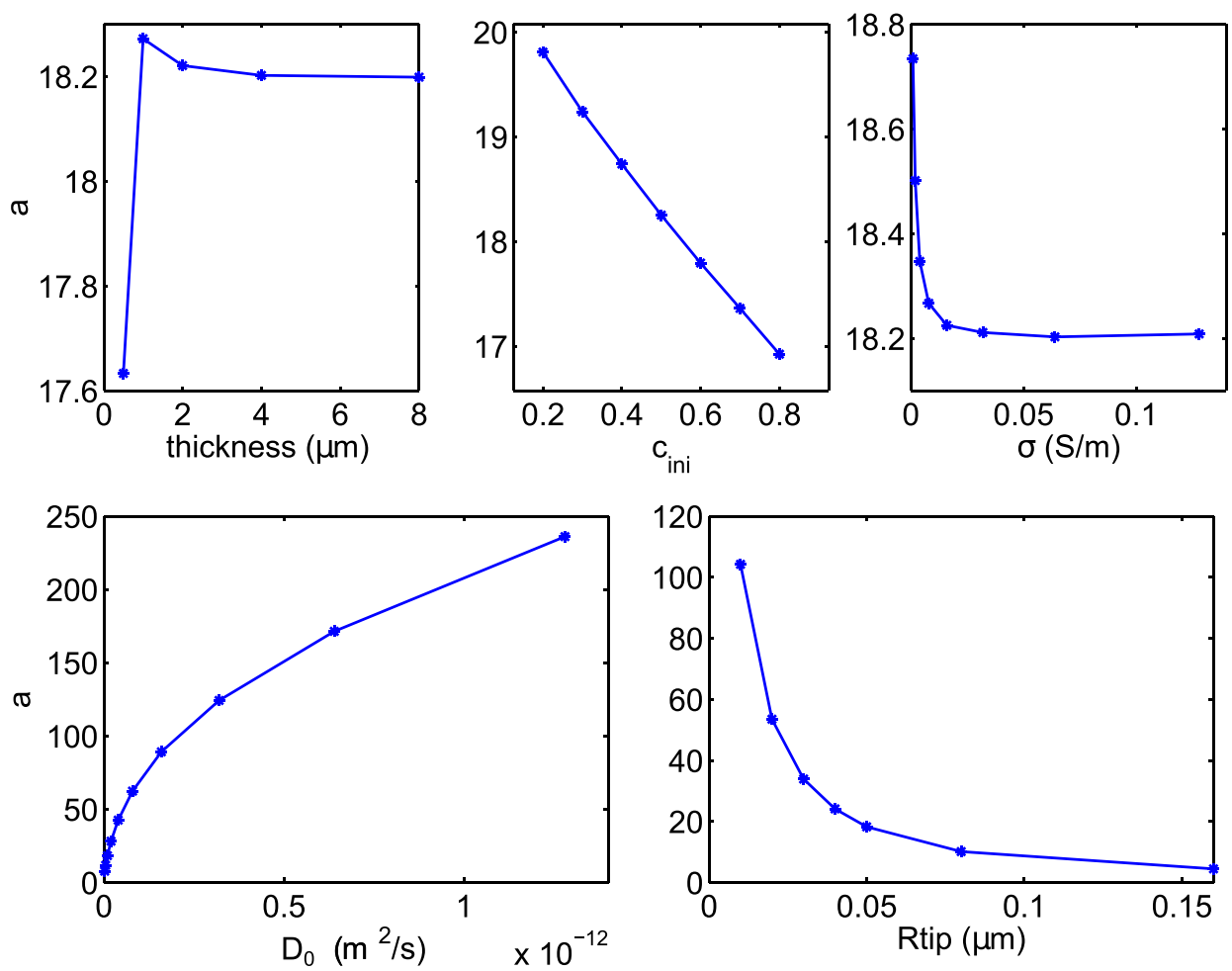

FIG. 13. Parameter study for $a$ with respect to the default state. Note that the $\mathrm{y}$ axis range is different for each plot for better visibility. A significant variation is only observed for $D_{0}$ and $R_{\text {tip }}$. dependent diffusion coefficient was implemented in order to simulate the three stable phases of $\mathrm{LiMn}_{2} \mathrm{O}_{4}$.

It was shown that the system tries to minimize the presence of intermediate concentrations. This leads to nonsymmetric hysteresis loops during voltage spectroscopy measurements. As it is difficult to extract useful information from this type of measurements, time spectroscopy measurements were considered. Using the phase field parameter, the signal is very dependent on concentration and applied DC voltage. A parametric study of time spectroscopy outputs was performed using a simpler model with constant diffusion coefficient. A power law of the form $(a t+1)^{1 / p}$ was found to fit the decay. It was shown that the decay of the signal is mainly dependent on the diffusion coefficient and the tip radius with $a \propto \sqrt{ } D_{0} / R_{\text {tip }}$.

To verify the modeling results, ESM measurements were carried out. Hysteresis loops show a diamond-like shape as predicted by the simulated loops. Butterfly like shapes were also observed. As this latter cannot be explained by the change of concentration under the tip, this could indicate that different mechanisms are taking place during ESM experiment. On the other hand, time spectroscopy measurements could be fitted with the power law, yielding a qualitative insight on the diffusion parameter. It is suggested that the

TABLE II. Fitting parameters of experimental measurements.

\begin{tabular}{lccc}
\hline \hline Sample & $a$ & $p$ & Coefficient of determination \\
\hline Fresh 0\%SoC & 2.69 & 0.45 & 0.9775 \\
Fresh 50\%SoC & 1.419 & 0.3234 & 0.9964 \\
Fresh 100\%SoC & 4.515 & 0.6846 & 0.9548 \\
Aged 0\%SoC & 0.3366 & 0.257 & 0.9862 \\
\hline \hline
\end{tabular}

diffusion coefficient of aged samples is two orders of magnitude lower than that of the fresh specimen. The value of the power exponent $p$ seems unrealistic considering the model results, hence quantitative conclusions should be taken with care. Three causes for the discrepancy are suggested: (1) in reality, the electrodes are not pure ion-blocking, (2) lithium diffusivity is dependent on the concentration as predicted by the phase model, and (3) ionic-polaronic coupling can take place.

The comparison of the simulations with ESM measurements undertaken in this work can serve as guidelines for further application of ESM on battery materials.

\section{ACKNOWLEDGMENTS}

This work and the $\mathrm{PhD}$ research of Amanieu, Luchkin, and Thai were supported by the European Commission within the FP7 Marie Curie Initial Training Network "Nanomotion" (Grant Agreement No. 290158, www.nanomotion.eu). We are also grateful for the support of the German public funded project ReLiOn (Contract No. 03X4619C), funded by the German Federal Ministry of Education and Research (BMBF) and managed by the Project Management Agency Forschungszentrum Jülich (PTJ). The authors wish to acknowledge CICECO. M.-A. K. acknowledges the financial support of the German Research Foundation (DFG) in the framework of the Cluster of Excellence in "Simulation Technology" EXC 310/2.

\footnotetext{
${ }^{1}$ M. Park, X. Zhang, M. Chung, G. B. Less, and A. M. Sastry, J. Power Sources 195, 7904 (2010).

${ }^{2}$ J. Vetter, P. Novak, M. Wagner, C. Veit, K. C. Muller, J. Besenhard, M. Winter, M. Wohlfahrt Mehrens, C. Vogler, and A. Hammouche, J. Power Sources 147, 269 (2005).
} 
${ }^{3}$ A. N. Morozovska, E. A. Eliseev, N. Balke, and S. V. Kalinin, J. Appl. Phys. 108, 053712 (2010).

${ }^{4}$ S. V. Kalinin and A. N. Morozovska, J. Electroceram. 32, 51 (2014).

${ }^{5}$ S. Guo, S. Jesse, S. Kalnaus, N. Balke, C. Daniel, and S. V. Kalinin, J. Electrochem. Soc. 158, A982 (2011).

${ }^{6}$ R. F. Mamin, I. K. Bdikin, and A. L. Kholkin, Appl. Phys. Lett. 94, 222901 (2009).

${ }^{7}$ K. Hoang, J. Mater. Chem. A 2, 18271 (2014).

${ }^{8}$ J. Guan and M. Liu, Solid State Ionics 110, 21 (1998).

${ }^{9}$ S. Jesse, N. Balke, E. Eliseev, A. Tselev, N. J. Dudney, A. N. Morozovska, and S. V. Kalinin, ACS Nano 5, 9682 (2011).

${ }^{10}$ A. N. Morozovska, E. A. Eliseev, and S. V. Kalinin, J. Appl. Phys. 111, 014114 (2012).

${ }^{11}$ A. Tselev, A. N. Morozovska, A. Udod, E. A. Eliseev, and S. V. Kalinin, Nanotechnology 25, 445701 (2014).

${ }^{12}$ R. A. Huggins, Advanced Batteries (Springer Verlag, 2009), Chap. 17, pp. 414431.

${ }^{13}$ N. Balke, S. Jesse, Y. Kim, L. Adamczyk, A. Tselev, I. Ivanov, N. Dudney, and S. Kalinin, Nano Lett. 10, 3420 (2010).

${ }^{14}$ S. Y. Luchkin, K. Romanyuk, M. Ivanov, and A. L. Kholkin, "Li transport in fresh and aged $\mathrm{LiMn}_{2} \mathrm{O}_{4}$ cathodes via electrochemical strain micro scopy," J. Appl. Phys. (to be published).

${ }^{15}$ C. Julien, A. Mauger, K. Zaghib, and H. Groult, Inorganics 2, 132 (2014).

${ }^{16} \mathrm{H}$. Berg and J. Thomas, Solid State Ionics 126, 227 (1999).

${ }^{17}$ C. Julien and M. Massot, Mater. Sci. Eng., B 100, 69 (2003).

${ }^{18}$ A. Van Der Ven, J. Bhattacharya, and A. Belak, Acc. Chem. Res. 46, 1216 (2013).

${ }^{19}$ E. Bohn, T. Eckl, M. Kamlah, and R. McMeeking, J. Electrochem. Soc. 160, A1638 (2013).

${ }^{20}$ D. W. Chung, N. Balke, S. V. Kalinin, and R. E. García, J. Electrochem. Soc. 158, A1083 (2011).

${ }^{21}$ H. Y. Amanieu, D. Rosato, M. Sebastiani, F. Massimi, and D. C. Lupascu, Mater. Sci. Eng., A 593, 92 (2014).
${ }^{22}$ A. Kumar, F. Ciucci, A. Morozovska, S. Kalinin, and S. Jesse, Nat. Chem. 3, 707 (2011).

${ }^{23}$ See supplemental material organized in four appendices at http://dx.doi.org/ 10.1063/1.4927747. Appendix A relates to the description of the Gibbs free energy. Appendix B relates to the description of $D_{\text {eff }}$ and $g$. Appendix C relates to butterfly like loops. Appendix D is additional figures and anima tions from experimental measurements and from the model.

${ }^{24} \mathrm{H}$. Dal and C. Miehe, Comput. Mech. 55, 303 (2015).

${ }^{25}$ A. R. Allnatt and A. B. Lidiard, Atomic Transport in Solids (Cambridge University Press, 1993).

${ }^{26}$ E. Iguchi, N. Nakamura, and A. Aoki, Philos. Mag. B 78, 65 (1998).

${ }^{27}$ E. Iguchi, Y. Tokuda, H. Nakatsugawa, and F. Munakata, J. Appl. Phys. 91, 2149 (2002)

${ }^{28}$ K. Tateishi, D. du Boulay, and N. Ishizawa, Appl. Phys. Lett. 84, 529 (2004).

${ }^{29}$ J. Marzec, S. Świerczek, J. Przewoźnik, J. Molenda, D. Simon, E. Kelder, and J. Schoonman, Solid State Ionics 146, 225 (2002).

${ }^{30} \mathrm{E}$. Bohn,"Partikel Modell fur Lithium Diffusion und mechanische Spannungen einer Interkalationselektrode," Ph.D. thesis (Karlsruher Institut fur Technologie, 2011).

${ }^{31}$ R. E. García, Y. M. Chiang, W. C. Carter, P. Limthongkul, and C. M. Bishop, J. Electrochem. Soc. 152, A255 (2005).

${ }^{32}$ B. M. Bolotovskiı̌ and A. V. Serov, Phys. Usp. 37, 515 (1994).

${ }^{33}$ S. Choudhury, J. X. Zhang, Y. L. Li, L. Q. Chen, Q. X. Jia, and S. V. Kalinin, Appl. Phys. Lett. 93, 162901 (2008).

${ }^{34}$ H. Y. Amanieu, M. Aramfard, D. Rosato, L. Batista, U. Rabe, and D. C. Lupascu, Acta Mater. 89, 153 (2015).

${ }^{35}$ Q. N. Chen, Y. Ou, F. Ma, and J. Li, Appl. Phys. Lett. 104, 242907 (2014).

${ }^{36}$ E. Deiss, Electrochim. Acta 47, 4027 (2002).

${ }^{37}$ X. C. Tang, X. W. Song, P. Z. Shen, and D. Z. Jia, Electrochim. Acta 50, 5581 (2005).

${ }^{38}$ A. N. Morozovska, E. A. Eliseev, S. L. Bravina, F. Ciucci, G. S. Svechnikov, L. Q. Chen, and S. V. Kalinin, J. Appl. Phys. 111, 014107 (2012).

${ }^{39}$ T. D. Nguyen, S. Mao, Y. W. Yeh, P. K. Purohit, and M. C. McAlpine, Adv. Mater. 25, 946 (2013). 